\title{
Efectos de las operaciones motivadoras en una conducta autolesiva mediante las presesiones
}

\section{Effects of motivating operations on self-harming behaviour through presessions}

\author{
David Simó-Pinatella ${ }^{1}$, Elisabeth Alomar-Kurz ${ }^{1}$, Josep Font-Roura ${ }^{2}$, \\ Climent Giné ${ }^{1}$ y Peter McGill ${ }^{3}$ \\ ${ }^{1}$ Facultat de Psicologia, Ciències de l'Educació i de l'Esport. Blanquerna. Universitat Ramon Llull. Barcelona. España. ${ }^{2}$ CPT \\ Estel. Vic. Barcelona. España. ${ }^{3}$ Tizard Centre. University of Kent. Canterbury. Reino Unido.
}

Disponible online 31 de diciembre de 2015

\begin{abstract}
En este estudio se exploran los efectos de las operaciones motivadoras sobre la ocurrencia de la conducta autolesiva que presenta una mujer de 42 años con discapacidad intelectual. El análisis funcional muestra que la conducta se mantiene por refuerzo social positivo (atención y tangible) y por refuerzo automático. Se ha utilizado un diseño múltiple en la intervención con el fin de explorar los efectos de las presesiones (acceso y no acceso a atención) en la frecuencia con la que se manifiesta dicha conducta. Los resultados obtenidos en la intervención muestran cómo la operación motivadora actúa como operación de abolición reduciendo la frecuencia con la que sucede la conducta analizada. El estudio concluye enfatizando en el uso de estrategias preventivas para evitar las conductas problemáticas, así como en la necesidad de realizar una evaluación funcional para comprender la naturaleza de las conductas problemáticas y diseñar planes de intervención que ayuden a minimizarlas.
\end{abstract}

Palabras Clave: Conductas Problemáticas; Análisis Funcional; Operaciones Motivadoras; Discapacidad Intelectual; Caso Clínico; Experimento de Caso Único.

This study explored the effects of motivating operations on the self-harming behaviour of a 42-year-old woman with intellectual disabilities. Functional analysis showed that her behaviour was maintained by positive social (attention and tangible) and automatic reinforcement. A multielement design was used to explore presession effects (access to attention and attention deprivation) on the frequency of the behaviour. The results showed that when access to attention was presented to the participant, the frequency of the behaviour decreased, suggesting the action of an abolishing operation. In conclusion, the use of preventive strategies to treat problem behaviours are emphasised, as well as the need for a functional assessment to understand the nature of these behaviours and to design intervention plans aimed at minimizing such behaviour.

Key Words: Problem Behaviour; Functional Analysis; Motivating Operations; Intellectual Disabilities; Clinical Case; Single Case Experiment.

Correspondencia: David Simó Pinatella. Facultat de Psicologia, Ciències de l'Educació i de l’Esport. Blanquerna. Universitat Ramon Llull. c/ Císter, 34.08022 Barcelona. E-mail: davidsp@blanquerna.url.edu. Fax: 932533031. E-mails de los co-autores: Elisabeth Alomar-Kurz: elisabethak@blanquerna.url.edu, Josep Font-Roura: j.font@santtomas.cat, Climent Giné: climentgg@blanquerna.url.edu, Peter McGill: p.mcgill@kent.ac.uk 
Se estima que la prevalencia de las conductas autolesivas entre las personas con discapacidad intelectual (DI) oscila entre el 10\% y 12\% (Sigafoos, O'Reilly, Lancioni, Lang y Didden, 2014; Van Ingen, Moore, Zaja, y Rojahn, 2010) y se considera que las conductas de golpearse en la cabeza con la mano o contra algún objeto son las más comunes (Sigafoos et al., 2014). Además, la prevalencia de estas conductas está relacionada con el grado de DI (Emerson, 2001; Sigafoos et al., 2014).

En general, dar respuesta a estas conductas, así como a otras tipologías de conductas problemáticas que presentan las personas con DI, es uno de los temas que más preocupa a los profesionales. Siguiendo las premisas del apoyo conductual positivo, es fundamental explorar aquellos eventos del entorno relacionados con la ocurrencia de estas conductas con el fin de crear entornos ecológicos y preventivos (Bambara y Knoster, 2009). En esta dirección, recientes investigaciones (por ejemplo, Butler y Luiselli, 2007; Cautilli y Dziewolska, 2004; Lanovaz, Fletcher y Rapp, 2009; Simó-Pinatella et al., 2013) evidencian la importancia de alterar o incidir en aquellos antecedentes -estímulos discriminativos y operaciones motivadoras (OM; Steege y Watson, 2009)- que propician la manifestación de conductas problemáticas (Miltenberg, 1998). Actualmente, se entiende que un estímulo discriminativo es aquel que precede a una conducta problemática y se señala la probabilidad de que se mantenga el refuerzo posterior a la conducta (Cooper, Heron y Heward, 2007; Steege y Watson, 2009). Por otro lado, las OM, término propuesto por Laraway, Snycerski, Michael y Poling (2003), son el resultado de la evolución de la terminología operante (Langthorne y McGill, 2009). Inicialmente, el concepto utilizado para referirse a aquellos antecedentes que alteraban tanto la efectividad de las consecuencias reforzantes (efecto establecedor-reforzante) como la frecuencia de las respuestas que habían sido asociadas a estas consecuencias en el pasado (efecto evocativo; Michael, 1982) era operaciones establecedoras. Sin embargo, la utilización de esta designación tenía limitaciones y no representaba los efectos bidireccionales de estos antecedentes (Langthorne y McGill, 2009); es decir, efectos establecedores (aumentar) y de abolición (disminuir). De este modo, Laraway et al. (2003) proponen la utilización de este nuevo concepto, OM, para referirse tanto a las operaciones establecedoras como a las de abolición. Así, se entiende que una $\mathrm{OM}$ es un evento que altera (a) la eficacia de un estímulo que actúa como reforzador y (b) la frecuencia actual de las conductas que han sido reforzadas por ese estímulo (Michael, 2007); es decir, las OM son antecedentes que tienen dos propiedades principales (Laraway et al., 2003; Laraway, Snycerski, Olson, Becker y Poling, 2014). La primera propiedad, el efecto de alterar el valor (value-altering effect), hace referencia al impacto que tiene un antecedente en la efectividad de otro estímulo que actúa como un tipo de refuerzo o castigo (Langthorne y McGill, 2009). La segunda propiedad, el efecto de alterar la conducta (behavior-altering effect), hace referencia al grado que tiene de alterar la probabilidad de que se manifieste una conducta que ha sido asociada a unas consecuencias en el pasado (Laraway et al., 2003).

Ciertamente, ha habido un creciente interés por explorar los efectos de las $\mathrm{OM}$ en el tratamiento de las conductas problemáticas de las personas con DI (Laraway et al., 2014; Luiselli, 2006). Los efectos de las OM en las conductas problemáticas se han estudiado en la literatura mediante las presesiones, especialmente en niños o adolescentes con DI (por ejemplo, Davis, Fuentes y Durand, 2014; O'Reilly et al., 2012; Rispoli et al., 2013); es decir, proporcionado acceso (o una modificación, o no acceso) a un evento que mantiene la conducta problemática antes de una situación referente (Simó-Pinatella et al., 2011). Por ejemplo, O'Reilly et al. (2007) implementaron dos condiciones de presesión para las conductas autolesivas y heteroagresivas, que se mantenían por acceso a tangible que presentaba un adolescente con autismo y DI. Las dos condiciones de la presesión planteadas por los investigadores eran: (a) acceso a tangible durante 15 minutos y (b) no acceso a tangible durante dos horas antes de la sesión control. Los resultados de este estudio sugieren que tener acceso a tangible actúa como operación de abolición disminuyendo la frecuencia con la que se manifiestan las conductas problemáticas, mientras que no proporcionar acceso a tangible actúa como operación establecedora aumentando su ocurrencia. Resultados parecidos a esta investigación se han obtenido en otros estudios en los que se ha proporcionado acceso -o no acceso- al refuerzo que mantienen las conductas problemáticas de los participantes (por ejemplo, Carter y Wheeler, 2007; Lang et al., 2009, 2010; McGinnis, Houchins-Juarez, McDaniel y Kennedy, 2010; O'Reilly, et al., 2008; Rapp, 2004; Roantree y Kennedy, 2006).

Un elemento que cabe destacar en cuanto a la eficacia de estas intervenciones recae en el conocimiento de aquellos refuerzos que mantienen las conductas problemáticas; es decir, es necesario comprender la naturaleza de las conductas problemáticas que presentan las personas con discapacidad desde su función y no desde su topografía (Arias, Irurtia y Gómez, 2009; Emerson y Einfeld, 2011). Preguntarse por el tipo de variables que influyen en la manifestación de las conductas problemáticas, así como por qué se realizan estas conductas, constituye una de las claves de cualquier intervención (Bambra y Knoster, 2009).

Una manera de comprender dichas conductas es mediante el análisis funcional que se ha convertido en el «estándar de oro» en el momento de identificar la función de la conducta problemática (Healy, Brett y Leader, 2013), pues permite demostrar empíricamente la relación causal entre las conductas problemáticas y los refuerzos que mantienen la conducta (Iwata y Dozier, 2008). El análisis funcional implica una manipulación experimental de aquellas variables que están relacionadas con la conducta objeto de estudio (Hanley, 2012; Nicholson, Konstantinidi y Furniss, 2006) y que proporcionan un refuerzo específico a la conducta de acuerdo con la condición experimental en la que se encuentra (Healy et al., 2013). El primer análisis 
funcional fue publicado por Iwata, Dorsey, Slifer, Bauman y Richman (1994); en este proponían aplicar esta metodología a la hora de evaluar las relaciones funcionales de las conductas autolesivas de nueve niños mediante cuatro condiciones: atención, demanda, solo y juego. No obstante, actualmente, las condiciones comunes que se utilizan en el momento de realizar un análisis funcional incorporan una quinta condición en la que se explora si la conducta problemática propicia acceso a un tangible preferido por el participante (Carr, LeBlanc y Love, 2009). Además, la investigación ha sugerido distintas variaciones de acuerdo con las necesidades conductuales de los participantes (Hanley, 2012; Iwata y Dozier, 2008).

Considerando, por un lado, la importancia del análisis funcional para comprender los refuerzos de las conductas problemáticas $\mathrm{y}$, por el otro, la evidencia de que modificando o alterando la motivación para presentar las conductas problemáticas se pueden disminuir dichas conductas, este estudio pretende explorar los efectos de las OM con una participante que presenta una conducta autolesiva. Los autores creen que si se manipulan las OM relacionadas con la conducta autolesiva, la ocurrencia de dicha conducta se verá alterada.

\section{Participante y entorno}

En este estudio ha participado una mujer de 42 años con DI severa y diagnosticada con el síndrome de fenilcetonuria. Esta participante manifiesta una conducta autolesiva que se concreta en golpearse la cabeza con la mano o contra la pared; como consecuencia de la autolesión, ha perdido la visión. La participante tiene habilidades comunicativas muy básicas y es capaz de responder a demandas sencillas que solicite el profesional. A su vez, a pesar de que se desplaza sola por los distintos espacios interiores del campus en el que reside, requiere de la presencia de una persona que la ayude a desplazarse por entornos desconocidos, pues muestra inseguridad e inestabilidad al andar. La participante recibe tratamiento farmacológico (por ej., tranxilium, arenbil y etumina) y atención individualizada una hora al día (centrada en estimulación sensorial y basal).

La participante vive en una residencia para personas con DI severa-profunda con necesidades de apoyo extenso y generalizado. Este servicio está constituida por 60 usuarios organizados en unidades familiares de 10 usuarios. Todas las sesiones del análisis y de la posterior intervención se realizaron en una sala de esta residencia conocida por la participante. La sala disponía de un sofá, una silla y los elementos necesarios para el estudio (ver secciones de instrumento y procedimiento).

\section{Instrumento}

El análisis funcional se realizó mediante un diseño multielemento, y el procedimiento fue parecido al descrito por Healy et al. (2013), se diseñaron cinco condiciones de análisis funcional: atención, evitación, tangible, sola y juego. No obstante, y de acuerdo con Iwata y Dozier (2008), se modificó la con- dición de atención y se realizó la variación de «atención dividida»; es decir, la profesional interactuaba con el investigador (Fahmie, Iwata, Harper y Querim, 2013) y solo se proporcionaba refuerzo verbal a la participante en el caso de manifestar la conducta objeto de estudio. Por otro lado, en la condición de demanda se le realizaban demandas sencillas relacionadas con los hábitos personales (por ejemplo, vestirse, sujetar y devolver un objeto a la profesional) y en caso de que la participante manifestara la conducta que se estaba estudiando, se interrumpía la actividad durante unos 30 segundos y luego se reanudaba. Además, considerando que la participante no tenía ninguna preferencia por un objeto físico en concreto, antes de iniciar la condición de tangible, se le ofrecían dos minutos de acceso a música. Este acceso se proporcionaba mediante un radiocasete que controlaba la profesional. Si durante la condición de evaluación la participante manifestaba la conducta problemática, se le proporcionaba acceso a música durante 30 segundos. En la condición de sola (o ignorar), aunque la participante estaba en el aula/taller con la profesional, no había interacción alguna entre ambas. Finalmente, en la condición de juego, la profesional proporcionaba atención continua a la participante y, a su vez, esta tenía acceso a música.

Todas las condiciones del análisis funcional tuvieron una duración de 10 minutos y se repitieron cinco veces de manera aleatoria. Las sesiones se grabaron en vídeo, y la frecuencia de la conducta autolesiva se puntuó mediante un registro de intervalos de 10 segundos. El primer y el segundo autor evaluaron independientemente el $40 \%$ de las sesiones. El acuerdo entre observador se calculó dividiendo el número de intervalos en que los observadores estaban de acuerdo por el número total de intervalos y multiplicando el resultado por 100. La media de acuerdo entre observadores fue del 96.99\% (rango, 91.66\% a $100 \%$ ).

\section{Procedimiento}

Una vez el proyecto fue aprobado por el comité de la universidad, los criterios de inclusión de la participante se centraron en (a) tener DI, (b) presentar una conducta problemática y (c) tener el consentimiento por parte de la familia o del tutor para participar en esta investigación. De acuerdo a estos criterios, la participante fue elegida por los profesionales del centro atendiendo la gravedad de su conducta problemática.

Antes de realizar el análisis funcional se entrevistó a una profesional de atención directa que conocía a la participante desde hacía más de seis meses, con el fin de definir la conducta problemática de manera precisa y, a su vez, identificar objetos o actividades preferidas para diseñar cuidadosamente el análisis funcional. A partir de esta entrevista se llegó a la conclusión de que la conducta problemática se mantenía por refuerzo social (atención y acceso a tangible) y refuerzo automático. Paralelamente, y como proponen Carter y Wheeler (2007), se realizó una observación por parte del investigador para evidenciar cómo se manifestaba la conducta descrita por la profesional. 


\section{OPERACIONES MOTIVADORAS Y CONDUCTAS PROBLEMÁTICAS}

Una vez realizado el análisis funcional, y con el fin de evaluar el impacto de las OM, se realizó un diseño multielemento en el que se alternaron dos condiciones de presesión: presesión de atención y presesión de no atención. En la primera condición, presesión de atención, se proporcionaba atención verbal continua a la participante $y$, a la vez, refuerzo físico de manera esporádica. En ningún momento, la profesional realizaba demandas a la participante. Además, y considerando que escuchar música era una actividad altamente preferida, se le proporcionaba acceso a música durante este tiempo. En el caso que se manifestara la conducta problemática, se dejaba de pro- momento en el que se registró la frecuencia de la conducta problemática. Todas las presesiones tuvieron una duración de 10 minutos. Con el fin de evitar posibles efectos de acumulación $\mathrm{u}$ otros efectos de las presesiones sobre la frecuencia de la conducta evaluada, se realizó una presesión al día y tres sesiones a la semana.

Del mismo modo que en el análisis funcional, todas las sesiones fueron grabadas y se realizó el acuerdo entre observadores para el $40 \%$ de las sesiones de la intervención. La media del acuerdo entre observadores fue del $95.99 \%$ (rango, 95\% a $96.66 \%$ ).

Figura 1

Intervalos en los que ocurre la conducta autolesiva a lo largo de las distintas condiciones del análisis funcional.

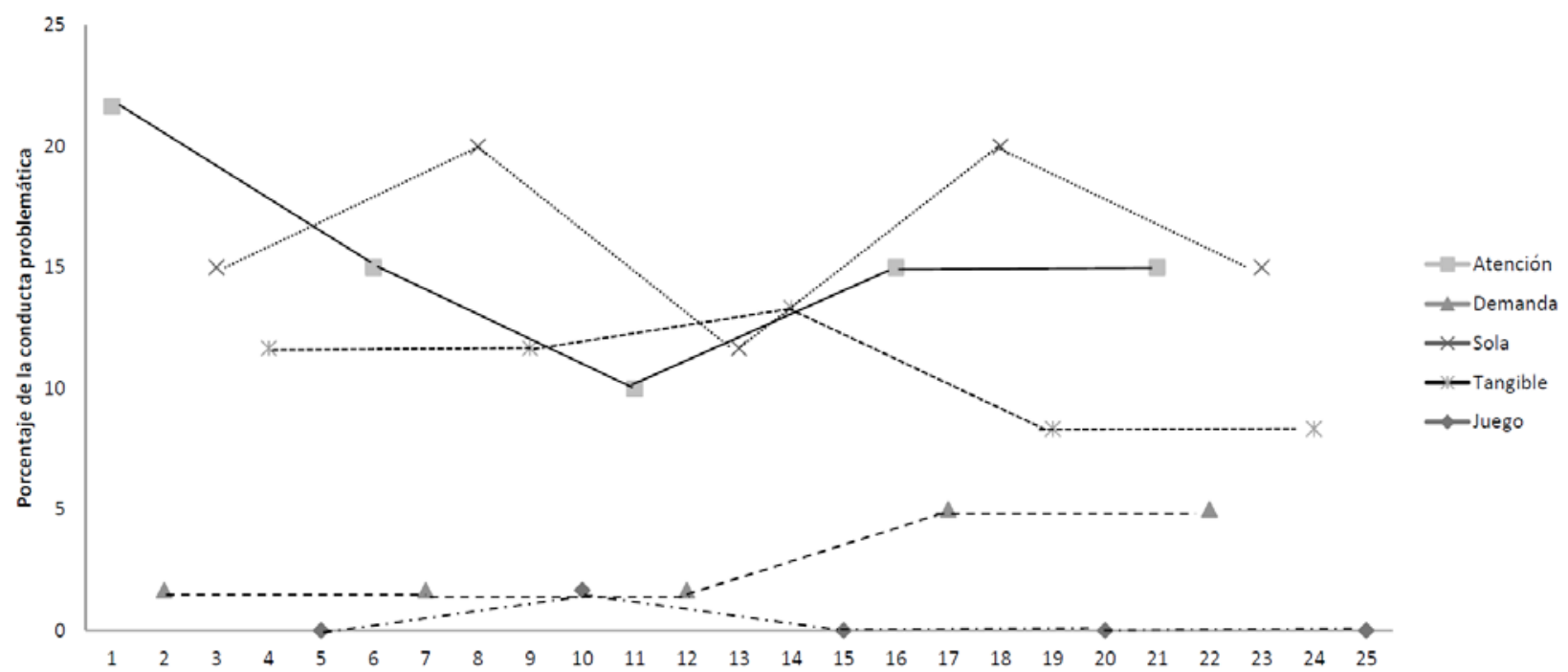

porcionar atención física y/o verbal por un período de 20-30 segundos. Por otro lado, en la condición de no atención, no se

Resultados proporcionaba ningún tipo de atención a la participante ni tenía acceso a ningún objeto/tangible. En el caso de que se produjera la conducta autolesiva, no se proporcionaba ninguna atención. Estas dos presesiones fueron seguidas de una condición control de atención (como la descrita en el análisis funcional);

Los resultados del análisis funcional se muestran en la figura 1. La participante ha manifestado la conducta autolesiva, con un mayor porcentaje de intervalos, en las condiciones en las que no se proporciona atención continua: atención $(M=15.66$, $D T=4.15)$, sola $(M=14.66, D T=3.62)$ y acceso a tangible $(M$ $=10.66, D T=2.24)$. Por lo contrario, en las condiciones en las

\section{Figura 2}

Intervalos en los que ocurre la conducta autolesiva a lo largo de las presesiones.

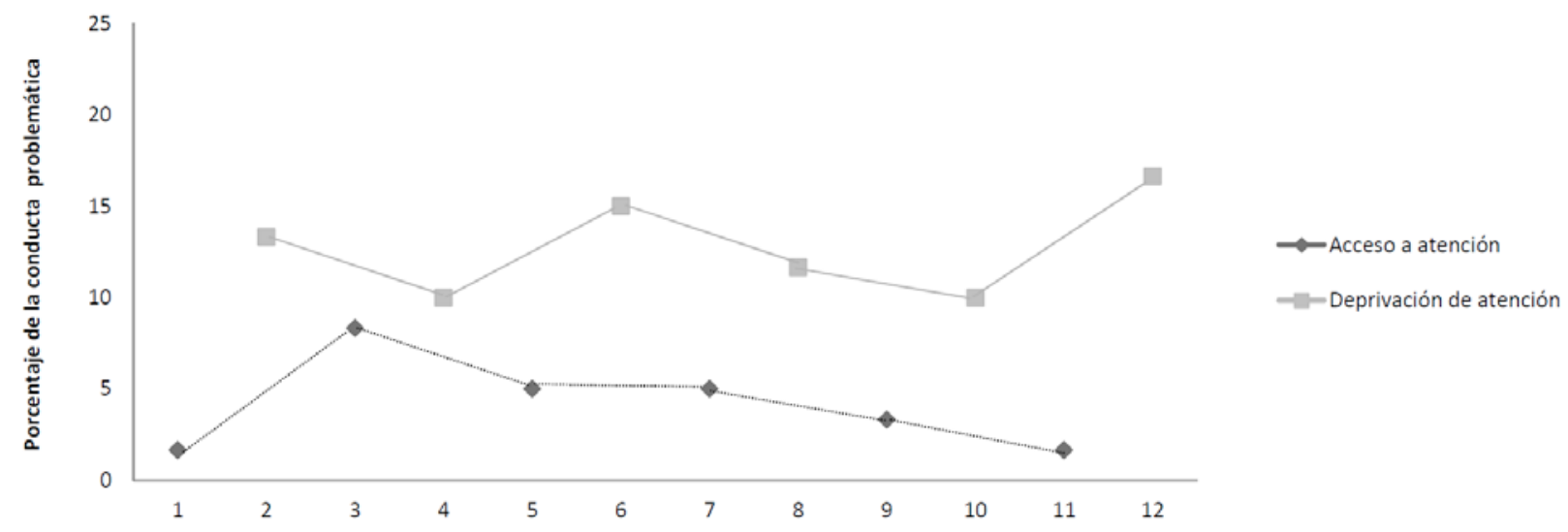


que se presenta una atención continua, la conducta problemática se produce con menor frecuencia (condición de demanda: $M=2.99, D T=1.83)$ o, en ocasiones, llega a no producirse durante el tiempo de evaluación (condición control/juego: $M=$ $0.33, D T=0.74)$.

Los resultados de la manipulación de las presesiones en la conducta problemática durante las sesiones de control se encuentran en la figura 2. En general, la frecuencia de la conducta problemática es menor cuando la condición de control ha ido precedida de una presesión en la que se ha ofrecido atención continua y música. Concretamente, en estos casos, el porcentaje de los intervalos en los que se manifiesta la conducta problemática oscila entre $1.66 \%$ y $8.33 \%(M=4.16, D T=2.53)$, mientras que aquellas condiciones de control en las que se ofrece una presesión sin acceso a atención por parte de la profesional dicha frecuencia oscila entre el $10 \%$ y el $16.66 \%(M=12.77, D T=$ 2.72) del total de los intervalos.

\section{Conclusiones}

El presente estudio se centra en explorar el efecto que determinadas OM provocan en la conducta autolesiva que presenta una persona con DI. Los resultados del análisis funcional sugieren que la frecuencia de la conducta evaluada está condicionada por el nivel de atención que recibe la participante; es decir, en aquellas condiciones en las que obtiene atención de manera continuada por parte del profesional (condiciones de juego y demanda), la frecuencia de la conducta es menor que en aquellas en las que no la recibe (condiciones de atención, sola y tangible). Por otro lado, atendiendo a los resultados obtenidos en la intervención, se observa que la atención actúa como OM; concretamente, recibir atención actúa como operación de abolición. Aun así, si comparamos los resultados del análisis funcional con los obtenidos en la intervención, se puede observar que el no recibir atención (en forma de presesión) no actúa como operación establecedora, pues estos no varían significativamente.

Distintas conclusiones se pueden extraer de este estudio. Primero, las intervenciones centradas en aquellos antecedentes relacionados con la ocurrencia de dichas conductas tienen un claro impacto en la propia conducta (Smith, 2011; Steege y Watson, 2009). Concretamente, las OM influyen de manera destacada en la manifestación de las conductas de las personas con DI. Es imperativo dedicar esfuerzos para identificar estos antecedentes con el fin de explorar hasta qué punto impactan en la conducta reduciendo o aumentando la frecuencia con la que esta conducta sucede (Simó-Pinatella et al., 2012). Aun así, y en el caso de este estudio, se ha podido comprobar que el no proporcionar atención no ha actuado como operación establecedora, pues, como señalan Chung y Cannella-Malone (2010), una OM puede tener distintos efectos en las personas que presentan las conductas. Segundo, el uso de las presesiones como estrategia para dar respuesta a las conductas problemáticas. En este sentido es fundamental explorar sus propiedades, así como el impacto que tienen en las conductas. Identificar las caracte- rísticas estructurales de las presesiones puede suponer dar un paso importante para los profesionales de atención directa a la hora de desarrollar planes de intervención preventivos (SimóPinatella et al., 2011). Además, los efectos de las presesiones pueden reducir la necesidad de intervenciones individualizadas en entornos ordinarios (Rispoli et al., 2011). Finalmente, la eficacia de las intervenciones depende en gran medida de si estas se ajustan a la funcionalidad de la conducta. Así, debemos comprender la complejidad de las conductas problemáticas contemplando aquellas variables que propician que ocurran, aquellas que las refuerzan, así como la relación entre ambas (Bambara y Knoster, 2009; Simó-Pinatella et al., 2013). Diseñar intervenciones que incorporen la función de la conducta (Ciponi y Schock, 2007) y actuar sobre los antecedentes permitirá desarrollar programas de intervención más educativos, preventivos y positivos (Dunlap y Carr, 2007; Sailor, Dunlap, Sugai y Horner, 2009).

Aunque los resultados de este estudio son claros, hay limitaciones que hay que mencionar. No se han recogido datos de mantenimiento o de generalización, pues no se obtienen datos de la frecuencia con la que se manifiesta la conducta problemática después de la intervención. De este modo, la investigación debería explorar los efectos de las $\mathrm{OM}$ en la generalización (O'Reilly et al., 2012). Asimismo, las variables relacionadas con la atención dividida no se han explorado, por ejemplo, el efecto del tipo de relación entre la profesional y la participante (Fahmie et al., 2013). Además, es necesario seguir explorando los efectos de estos antecedentes en otros participantes y con conductas distintas (O'Reilly et al., 2007), así como en contextos ordinarios.

Uno de los mayores retos que afrontan las organizaciones que atienden a personas con DI recae en el hecho de tener que crear entornos que se adecúen a las necesidades conductuales que manifiestan las personas; es decir, en identificar y modificar aquellas variables contextuales que influyen en la aparición de las conductas problemáticas y hacer cambios ecológicos. Así, es preciso proporcionar un entorno activo que evite la inactividad de las personas con DI e incite a la participación significativa (Beadle-Brown, Hutchinson y Whelton, 2012; Koritsas, Iacono, Hamilton y Leighton, 2008). De acuerdo con los resultados de este estudio, y atendiendo que la conducta autolesiva de la participante parece mantenerse por atención, sería necesario plantear cómo se puede dar este refuerzo de manera preventiva a lo largo del día o bien reforzando aquellos momentos en que dicha conducta se da con menor frecuencia. Evidentemente, este procedimiento no eliminará la conducta problemática, pero sí reducirá las probabilidades de que esta se produzca (Mansell y Beadle-Brown, 2012).

\section{Referencias}

1. Arias, B., Irurtia, M. J. y Gómez, L. E. (2009). Conductas problemáticas en personas con discapacidad intelectual: un estudio preliminar sobre dimensionalidad y propuesta de clasificación. Psicología Conductual, 17, 257-275. 
2. Bambara, L. M. y Knoster, T. P. (2009). Designing Positive Behavior Support Plans (2a ed.). Washington: AAIDD.

3. Beadle-Brown, J., Hutchinson, A. y Whelton, B. (2012). Person-Centred Active Support-Choice, Promoting Independence and Reducing Challenging Behaviour. Journal of Applied Research in Intellectual Disabilities, 25, 291-307. http://dx.doi.org/10.1111/j.1468-3148.2011.00666.x

4. Butler, L. R. y Luiselli, J. K. (2007). Escape-maintained problem behavior in a child with autism: Antecedet functional analysis and intervention evaluation of noncontingent escape and instructional fading. Journal of Positive Behavior Interventions, 9, 195-202. http://dx.doi.org/10.1177/10 983007070090040201

5. Carr, J. E., LeBlanc, L. A. y Love, J. r. (2009) Experimental functional analysis of problema behavior. En W. T. O'donohue y J.E. Fisher (Eds.), Cognitive Therapy: Applying empirically supported techniques in your practice, $2^{\mathrm{a}}$ edición, (pp. 211-219). Hoboken, NJ: Wiley.

6. Carter, S. L. y Wheeler, J. J. (2007). Analysis of behavioural responding across multiple instructional conditions for a child with childhood disintegrative disorder. Journal of Research in Special Educational Needs, 7, 137-141. http:// dx.doi.org/10.1111/j.1471-3802.2007.00090.x

7. Cautilli, J. y Dziewolska, H. (2004). A brief report: The neutralizing effects of stimulus control intervention for sleep on escape behavior and token performance of a nineyear-old child with oppositional defiant disorder. Journal of Early and Intensive Behavior Intervention, 1, 232-238. http://dx.doi.org/10.1037/h0100291

8. Chung, Y. C. y Cannella-Malone, H. I. (2010). The effects of presession manipulations on automatically maintained challenging behavior and task responding. Behavior Modification, 34, 479-502. http://dx.doi. org/10.1177/0145445510378380

9. Ciponi, E. y Schock, K.M. (2007). Functional behavioral assessment, diagnosis and treatment. A complete system for education and mental health. New York: Springer Publishing Company.

10. Cooper, J. O., Heron, T. E. y Heward, W. L. (2007). Applied behavior analysis ( 2 a ed.). Upper Saddle River, NJ: Pearson Education Inc.

11. Davis, T. N., Fuentes, L. y Durand, S. (2014). Examination of systematic durations of presession reinforcer access on functional communication training. Journal of Developmental and Physical Disabilities, 26, 263-270. http:// dx.doi.org/10.1007/s10882-013-9360-2

12. Dunlap, G. y Carr, E.G. (2007). Positive behavior support and developmental disabilities: a summary and analysis of research. En S.L. Odom, R. Horner, M. Snell y J. Blacher (Eds.), Handbook of developmental disabilities (pp. 469482). New York: The Guilford Press.

13. Emerson, E. (2001). Challenging behaviour. Analysis and intervention in people with severe intellectual disabili- ties (2nd ed.). Cambridge: University Press. http://dx.doi. org/10.1017/CBO9780511543739

14. Emerson, E. y Einfeld, S. T. (2011). Challenging behavior (3rd ed.). Cambridge, UK: Cambridge University Press. http://dx.doi.org/10.1017/CBO9780511861178

15. Fahmie, T. A., Iwata, B. A., Harper, J. M. y Querim, A. C. (2013). Evaluation of the divided attention condition during functional analyses. Journal of Applied Behavior Analysis, 46, 71-78. http://dx.doi.org/10.1002/jaba.20

16. Hanley, G.P. (2012). Functional assessment of problem behavior: Dispelling myths, overcoming implementation obstacles, and developing new lore. Behavior Analysis in Practice, 5, 54-72.

17. Healy, O., Brett, D. y Leader, G. (2013). A comparison of experimental functional analysis and the Questions About Behavioral Function (QABF) in the assessment of challenging behavior of individuals with autism. Research in Autism Spectrum Disorders, 7, 66-81. http://dx.doi. org/10.1016/j.rasd.2012.05.006

18. Iwata, B. A. y Dozier, C. L. (2008). Clinical application of functional analysis methodology. Behavior Analysis in Practice, 1, 3-9.

19. Iwata, B. A., Dorsey, M. F., Slifer, K. J., Bauman, K. E. y Richman, G. S. (1994). Toward a functional analysis of self-injury. Journal of Applied Behavior Analysis, 27, 197209. http://dx.doi.org/10.1901/jaba.1994.27-197

20. Koritsas, S., Iacono, T., Hamilton, D. y Leighton, D. (2008). The effect of active support training on engagement, opportunities for choice, challenging behaviour and support needs. Journal of Intellectual and Developmental Disability, 33, 247-256. http://dx.doi. org/10.1080/13668250802282944

21. Lang, R., O'Reilly, M., Sigafoos, M., Lancioni, G. E., Machalicek, W., Rispoli, M. y White, P. (2009). Enhancing the effectiveness of a play intervention by abolishing the reinforcing value of stereotypy: A pilot study. Journal of Applied Behavior Analysis, 42, 889-894. http://dx.doi. org/10.1901/jaba.2009.42-889

22. Lang, R., O’Reilly, M., Sigafoos, M., Machalicek, W., Rispoli, M., Lancioni, G. E., ... Fragale, C. (2010). The effects of an abolishing operation intervention component on play skills, challenging behavior and stereotypy. Behavior Modification, 34, 267-289. http://dx.doi. org/10.1177/0145445510370713

23. Langthorne, P. y McGill, P (2009). A Tutorial on the Concept of the Motivating Operation and its Importance to Application. Behavior Analysis in Practice 2, 22-31.

24. Lanovaz, M. J., Fletcher, S. E. y Rapp, J. T. (2009). Identifying stimuli that alter immediate and subsequent levels of vocal stereotypy: A further analysis of functionally matched stimulation. Behavior Modification, 33, 682-704. http://dx.doi.org/10.1177/0145445509344972

25. Laraway, S., Snycerski, S., Michael, J. y Poling, A. (2003). 
Motivating operations and terms to describe them: Some further refinements. Journal of Applied Behavior Analysis, 36, 407-414. http://dx.doi.org/10.1901/jaba.2003.36-407

26. Laraway, S., Snycerski, S., Olson, R., Becker, B. y Poling, A. (2014). The motivating operations concept: current status and critical response. The Psychological Record, 64, 601-623. http://dx.doi.org/10.1007/s40732-014-0080-5

27. Luiselli, J. K. (2006). Antecedent assessment and intervention. Supporting children and adults with developmental disabilities in community settings. Baltimore: Paul H Brookes.

28. Mansell, J. y Beadle-Brown, J. (2012). Active support: Enabling and empowering people with intellectual disabilities. Jessica Kingsley Publishers: Great Britain.

29. McGinnis, M. A., Houchins-Juarez, N., McDaniel, J. L. y Kennedy, C. H. (2010). Abolishing and establishing operation analyses of social attention as positive reinforcement for problem behavior. Journal of Applied Behavior Analysis, 43, 119-123. http://dx.doi.org/10.1901/jaba.2010.43$\underline{119}$

30. Michael, J. (1982). Distinguishing between discriminative and motivational funcions of stimuli. Journal of the Experimental Analysis of Behavior, 37, 149-155. http://dx.doi. org/10.1901/jeab.1982.37-149

31. Michael, J. (2007). Motivating operations. In J. O. Cooper, T. E. Heron y W. L. Heward (Eds.), Applied Behavior Analysis, 2nd ed., (pp. 374-391). Upper Saddle River, NJ: Pearson Education.

32. Miltenberg, R. G. (1998). Methods for Assessing Antecedent Influences on Challenging Behaviors. En J. K. Luiselli y M. J. Cameron (Eds.), Antecedent Control. Innovative Approaches to Behavioral Support (pp. 47-65). Baltimore: Paul H. Brookes Publishing Co.

33. Nicholson, J., Konstantinidi, E. y Fureniss, F. (2006). On some psychometric properties of the Questions About Behavioral Function (QABF) scale. Research in Developmental Disabilities, 27, 337-352. http://dx.doi.org/10.1016/j. ridd.2005.04.001

34. O'Rreilly, M., Aguilar, J., Fragale, C., Lang, R., Edrisinha, C., Sigafoos, J., ... Didden, R. (2012). Effects of a motivating operation manipulation on the maintenance of mands. Journal of Applied Behavior Analysis, 45, 443-447. http://dx.doi.org/10.1901/jaba.2012.45-443

35. O'Reilly, M., Edrisinha, C., Sigafoos, J., Lancioni, G., Cannella, H., Machalicek, W. y Langathorne, P. (2007). Manipulating the evocative and abative effects of an establishing operation: Influences on challenging behavior during classroom instruction. Behavioral Interventions, 22, 137-145. http://dx.doi.org/10.1002/bin.226

36. O'Reilly, M. F., Sigafoos, J., Lancioni, G., Rispoli, M., Lang, R., Chan, J., ... Langthorne, P. (2008). Manipulating the behavior-altering effect of the motivating operation: Examination of the influence on challenging beha- vior during leisure activities. Research in Developmental Disabilities, 29, 333-340. http://dx.doi.org/10.1016/j. ridd.2007.06.004

37. Rapp, J. T. (2004). Effects of prior access and environmental enrichment on stereotypy. Behavioral Interventions, 19, 287-295. http://dx.doi.org/10.1002/bin.166

38. Rispoli, M., Camargo, S. H., Neely, L., Gerow, S., Lang, R., Goodwyn, F. y Ninci, J. (2013). Pre-Session Satiation as a Treatment for Stereotypy During Group Activities. Behavior Modification, 38, 392-411. http://dx.doi. org/10.1177/0145445513511631

39. Rispoli, M. J., O’Reilly, M. F., Sigafoos, J., Lang, R., Kang, S., Lancioni, G. y Parker, R. (2011). Effects of presession satiation on challenging behavior and academic engagement for children with autism during classroom instruction.Education and Training in Autism and Developmental Disabilities, 46, 607-618.

40. Roantree, C. y Kennedy, C. H. (2006). A paradoxical effect of presession attention on stereotypy: Antecedent attention as an establishing, not an abolishing, operation. Journal of Applied Behavior Analysis, 39, 381-384. http://dx.doi. org/10.1901/jaba.2006.97-05

41. Sailor, W., Dunlap, G., Sugai, G., y Horner, R. (2009). Handbook of positive behavior support. New York: Springer. http://dx.doi.org/10.1007/978-0-387-09632-2

42. Sigafoos, J., O’Reilly, M. F., Lancioni, G. E., Lang, R. y Didden, R. (2014). Self-Injurious Behavior. En P. Sturmey y R. Didden, R. (Eds.). Evidence-based practice and intellectual disabilities (pp.133-162). Oxford: Wiley Blackwell. http://dx.doi.org/10.1002/9781118326077.ch6

43. Simó-Pinatella, D., Alomar-Kurz, E., Font-Roura, J., Giné, C., Planella-Morató, J. y McGill, P. (2011). Las presesiones como estrategia para tratar las conductas problemáticas de los alumnos con discapacidad intelectual: una revisión. Análisis y Modificación de Conducta, 37, 155156.

44. Simó-Pinatella, D., Font-Roura, J., Alomar-Kurz, E., Gine, C., Matson, J. L. y Cifre, I. (2013). Antecedent events as predictive variables for behavioral function.Research in Developmental Disabilities, 34, 4582-4590. http://dx.doi. org/10.1016/j.ridd.2013.09.040

45. Simó-Pinatella, D., Font-Roura, J., Planella-Morató, J., McGill, P., Alomar-Kurz, E. y Giné, C. (2012). Types of motivating operations in interventions with problem behavior: A systematic review. Behavior Modification, 37, 3-38. http://dx.doi.org/10.1177/0145445512448096

46. Smith, R. G. (2011). Developing Antecedent Interventions for Problem Behavior. En W. W. Fisher, C.C. Piazza y H. S. Roane (Eds.), Handbook of Applied Behavior Analysis (pp. 297-316). New York: The Guilford Press.

47. Steege, M. W. y Watson, T. S. (2009). Conducting schoolbased functional behavioral assessment. A practitioner's guide. 2nd edition. New York: Guilford. 
48. Van Ingen, D. J., Moore, L. L., Zaja, R. H. y Rojahn, J. (2010). The Behavior Problems Inventory (BPI-01) in community-based adults with intellectual disabilities: Reliability and concurrent validity vis-à-vis the Inventory for Client and Agency Planning (ICAP). Research in Developmental Disabilities, 31, 97-107. http://dx.doi.org/10.1016/j. ridd.2009.08.004

Fecha de recepción: 25 de marzo, 2015

Fecha de recepción de la versión modificada: 1 de julio, 2015

Fecha de aceptación: 1 de julio, 2015 